CARDIOVASCULAR MEDICINE

\title{
Post-challenge hyperinsulinaemia rather than hyperglycaemia is associated with the severity of coronary artery disease in patients without a previous diagnosis of diabetes mellitus
}

\author{
H Satoh, H Terada, A Uehara, H Katoh, M Matsunaga, K Yamazaki, F Matoh, H Hayashi
}

Heart 2005;91:731-736. doi: 10.1136/hrt.2003.032326

See end of article for authors' affiliations

Correspondence to:

Dr Hiroshi Satoh, Division

of Cardiology, Internal

Medicine III, Hamamatsu

University School of

Medicine, 1-20-1

Handayama, Hamamatsu,

431-3192, Japan;

satoh36@hama-med.ac.jp

Accepted 27 July 2004

\begin{abstract}
Objective: To ascertain the prevalence of abnormal glucose metabolism in patients with coronary artery disease (CAD) but no previous diagnosis of diabetes mellitus (DM) and to examine the relation between the severity of CAD and responses of glucose and insulin to the glucose tolerance test.

Methods and results: Abnormalities of glucose metabolism and insulin response were analysed in 144 patients with CAD without a previous diagnosis of $D M$ who underwent both coronary arteriography and $75 \mathrm{~g}$ oral glucose tolerance test. The proportions of impaired and diabetic glucose tolerance were very high (39\% for impaired and $21 \%$ for diabetic glucose tolerance); only $40 \%$ had normal glucose tolerance. The parameters of glucose metabolism were not associated with the number of diseased coronary arteries or the presence of previous myocardial infarction (MI). However, the insulin concentration at 60 minutes or 120 minutes after glucose challenge, insulin area, and the ratio of insulin to glucose area were significantly higher in patients with significant coronary stenosis and with previous MI. Fasting glucose concentration and most conventional risk factors did not predict post-challenge hyperinsulinaemia.

Conclusion: Patients with CAD without a previous diagnosis of DM had a high prevalence of abnormal glucose tolerance. Post-challenge hyperinsulinaemia was associated with the number of diseased coronary arteries and the presence of previous MI. The insulin response to the glucose challenge test requires further investigation as a potential risk factor for CAD and a potential target for intervention.
\end{abstract}

$\mathrm{T}$ he risk of diabetes mellitus (DM) for coronary artery disease (CAD) is now widely accepted. Several large cohort studies including DECODE (diabetes epidemiology: collaborative analysis of diagnostic criteria in Europe) have indicated that people with impaired glucose tolerance have a raised risk of cardiovascular morbidity and mortality, and that a plasma glucose (PG) concentration even below the threshold for DM is associated with CAD. ${ }^{1-3}$

Stabilisation of glucose concentrations in patients with DM can reduce microvascular complications, but whether such treatment can also prevent macrovascular complications remains undefined. Several studies showed that intensive blood glucose control with sulfonylureas or insulin had only a borderline effect on reduction of myocardial infarction (MI). ${ }^{4}$ Thus, the risk of CAD may have developed before the onset of overt DM. Recently, epidemiological studies have suggested that insulin resistance with hyperinsulinaemia is an important risk factor for CAD and for non-insulin dependent DM. Insulin resistance with hyperinsulinaemia is thought to promote atherosclerosis through the direct effects of insulin on arterial walls or through the associated metabolic abnormalities, such as hypertension, hyperglycaemia, and dyslipidaemia. ${ }^{5-8}$

However, there are few reports regarding the association between the severity of CAD and abnormal glucose metabolism and hyperinsulinaemia in view of secondary prevention. ${ }^{9}{ }^{10}$ The purpose of this study was to ascertain the prevalence of abnormal glucose metabolism in patients with CAD but no previous diagnosis of DM, and to examine the relation between the severity of $\mathrm{CAD}$ and responses of glucose and insulin to the glucose challenge test.

\section{METHODS}

\section{Patients}

We selected patients aged below 75 years who underwent both coronary arteriography for CAD and $75 \mathrm{~g}$ oral glucose tolerance test (75g-oGTT) at Hamamatsu University Hospital from 1998 to 2002. Patients being treated for DM, fasting PG concentration $\geqslant 7.0 \mathrm{mmol} / \mathrm{l}$, or haemoglobin $\mathrm{A}_{1 \mathrm{c}} \geqslant 6.5 \%$ were considered to have DM and were not selected. Patients with hypertrophic or dilated cardiomyopathies, valvar heart diseases, chronic renal failure (serum creatinine $>176.8 \mu \mathrm{mol} / \mathrm{l}$ ), and prior coronary revascularisations were also excluded. Thus, 144 patients without a previous diagnosis of DM were selected retrospectively. Twenty nine patients had vasospastic angina pectoris (that is, they were positive for acetylcholine evoked test), 32 had effort angina pectoris (20 patients were positive for stress myocardial perfusion scintigraphy, and seven patients had a positive exercise treadmill test, three patients had typical chest pain, and two patients had an ECG abnormality), 20 had an old MI, and 63 had acute coronary syndrome (29 unstable angina pectoris and 34 acute MI). Patients with stable angina pectoris or with an old MI underwent the $75 \mathrm{~g}$-oGTT within two weeks before or after coronary arteriography. Patients with acute coronary syndrome underwent the test between

Abbreviations: $75 \mathrm{~g}-\circ \mathrm{GT}, 75 \mathrm{~g}$ oral glucose tolerance test; AUCglu, area under the glucose curve; AUCins, area under the insulin curve; BMI, body mass index; $C A D$, coronary artery disease; $D E C O D E$, diabetes epidemiology: collaborative analysis of diagnostic criteria in Europe; DM, diabetes mellitus; HOMA-IR, insulin resistance estimated by homeostasis model assessment; IRI, plasma insulin; MI, myocardial infarction; PG, plasma glucose; VD, vessel disease 
Table 1 Basic characteristics of patients categorised according to the number of significantly stenosed coronary arteries

\begin{tabular}{llll}
\hline & 0-VD & 1-VD & $\geqslant 2$-VD \\
\hline Age (years) & $59.4(9.1)$ & $62.6(9.0)$ & $62.3(7.6)$ \\
Sex (male/female) & $21 / 10$ & $52 / 13$ & $42 / 6$ \\
MI (-) & $29 /$ & $31 /$ & $29 /$ \\
MI (+) & 2 & $34^{*}$ & $19^{*}$ \\
BMI (kg/m $\left./ \mathrm{m}^{2}\right)$ & $22.9(2.7)$ & $23.3(2.9)$ & $24.1(3.0)$ \\
Current smoker & $54.8 \%$ & $40.0 \%$ & $45.8 \%$ \\
$\mathrm{HbA} \mathrm{Ic}_{\mathrm{c}}(\%)$ & $5.5(0.5)$ & $5.6(0.4)$ & $5.4(0.4)$ \\
$\mathrm{TC}(\mathrm{mmol} / \mathrm{l})$ & $5.17(0.91)$ & $5.34(0.93)$ & $5.25(1.03)$ \\
$\mathrm{TG}(\mathrm{mmol} / \mathrm{ll})$ & $1.64(1.17)$ & $1.64(1.01)$ & $1.54(0.64)$ \\
$\mathrm{HDL}-\mathrm{C}(\mathrm{mmol} / \mathrm{l})$ & $1.37(0.39)$ & $1.28(0.32)$ & $1.14(0.28)^{*} \dagger$ \\
$\mathrm{SBP}(\mathrm{mm} \mathrm{Hg})$ & $132.4(14.6) 133.9(26.8) 133.5(19.0)$ \\
DBP (mm Hg) & $74.5(10.5)$ & $73.7(16.4)$ & $77.6(11.2)$ \\
Medications & & & \\
Calcium channel blockers & $96.8 \%$ & $56.3 \%^{*}$ & $70.8 \%^{*}$ \\
$\beta$ Adrenergic blockers & $0 \%$ & $60.0 \% \%^{*}$ & $62.5 \%^{*}$ \\
ACEI/ARB & $6.5 \%$ & $48.4 \%^{*}$ & $47.9 \%^{*}$ \\
Diuretics & $0 \%$ & $1.6 \%$ & $10.4 \%^{*} \dagger$ \\
Lipid lowering agents & $35.5 \%$ & $56.3 \%$ & $54.2 \%$ \\
\hline
\end{tabular}

Data are mean (SD).

${ }^{*} \mathrm{p}<0.05 \vee 0-V D ;+\mathrm{p}<0.05 \vee 1-\mathrm{VD}$ by $\chi^{2}$ test or Kruskal-Wallis test followed by Mann-Whitney $U$ test with Bonferroni correction. $A C E I$, angiotensin converting enzyme inhibitors; $A R B$, angiotensin II receptor blockers; $B M I$, body mass index; DBP, diastolic blood pressure; $\mathrm{HbA}{ }_{1 c}$, haemoglobin $\mathrm{A}_{1 c ;} \mathrm{HDL}-\mathrm{C}$, high density lipoprotein cholesterol; $\mathrm{Ml}$ $(-)$, no previous myocardial infarction; $M I(+)$, previous myocardial infarction; SBP, systolic blood pressure; TC, total cholesterol; TG, triglyceride; $\mathrm{VD}$, vessel disease.

2-3 weeks after the last coronary episode to avoid the possible influence on glucose tolerance.

\section{Blood samples and measurements}

Blood samples were collected after an overnight fast during hospital stay for the measurement of $P G$, plasma insulin
(IRI), serum lipids, and haemoglobin $\mathrm{A}_{\mathrm{lc}}$. PG concentration was measured by the glucose oxidase method and IRI was measured by standard radioimmunoassay. Both PG and IRI were measured before (PG-0, IRI-0) and 30 minutes (PG-30, IRI-30), 60 minutes (PG-60, IRI-60), and 120 minutes (PG120, IRI-120) after the ingestion of a $75 \mathrm{~g}$ oral glucose challenge. The total integrated PG and IRI responses were quantified by calculating the PG and IRI areas under the curve by use of the trapezoidal method (AUCglu and AUCins, respectively). Insulin resistance was also estimated by a homeostasis model assessment (HOMA-IR). ${ }^{11}$

\section{Coronary arteriography}

Coronary arteriography was performed by transfemoral or transradial approach with 4-6 French catheters. Luminal narrowing was defined with quantitative coronary analysis (Coronary, version 1.5.3.1, Elk Co, Japan). Stenosed vessels with $>50 \%$ luminal narrowing in the main coronary arteries or major branches were counted.

\section{Patient classification}

All patients were categorised by the results of $75 \mathrm{~g}$-oGTT according to the diagnostic thresholds of the Expert Committee on the Diagnosis and Classification of Diabetes Mellitus. ${ }^{12}$ Firstly, patients with fasting $\mathrm{PG}<100 \mathrm{mg} / \mathrm{dl}$ $(5.6 \mathrm{mmol} / \mathrm{l})$ and $100-125 \mathrm{mg} / \mathrm{dl} \quad(5.6-6.9 \mathrm{mmol} / \mathrm{l})$ were considered to have normal and impaired fasting glucose, respectively. Secondly, patients with PG at 120 minutes $<140 \mathrm{mg} / \mathrm{dl}$ (7.8 mmol/l), $140-199 \mathrm{mg} / \mathrm{dl}$ (7.8-11.0 mmol/ $1)$, and $\geqslant 200 \mathrm{mg} / \mathrm{dl}(11.1 \mathrm{mmol} / \mathrm{l})$ were considered to have normal, impaired, and diabetic glucose tolerance, respectively. The patients were also classified according to the coronary arteriography findings into three groups: those who had no significantly stenosed vessels ( 0 vessel disease (VD)), one significantly stenosed vessel (l-VD), and two or more stenosed vessels $(\geqslant 2-\mathrm{VD})$. The 0 -VD group comprised 29
A
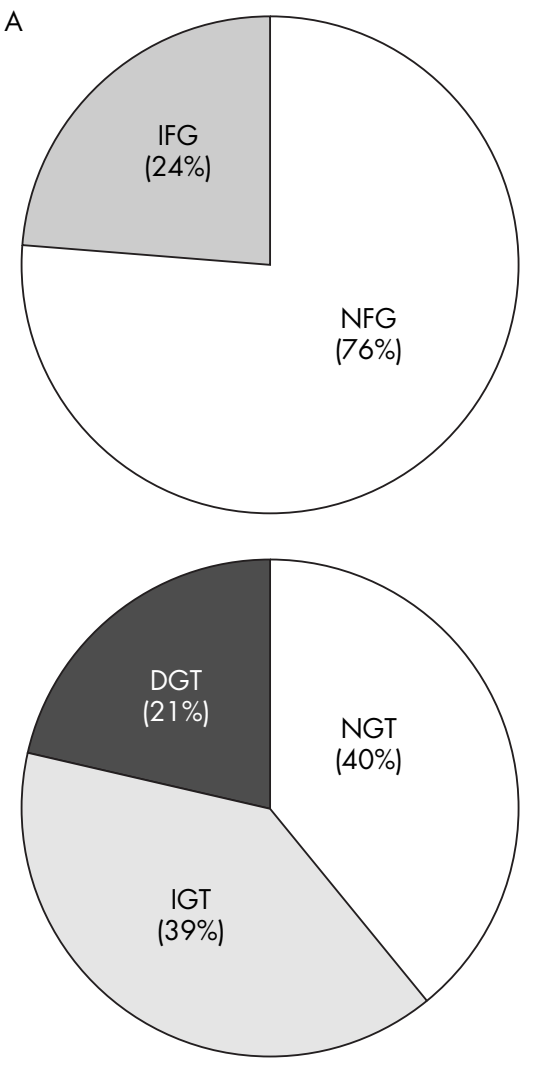
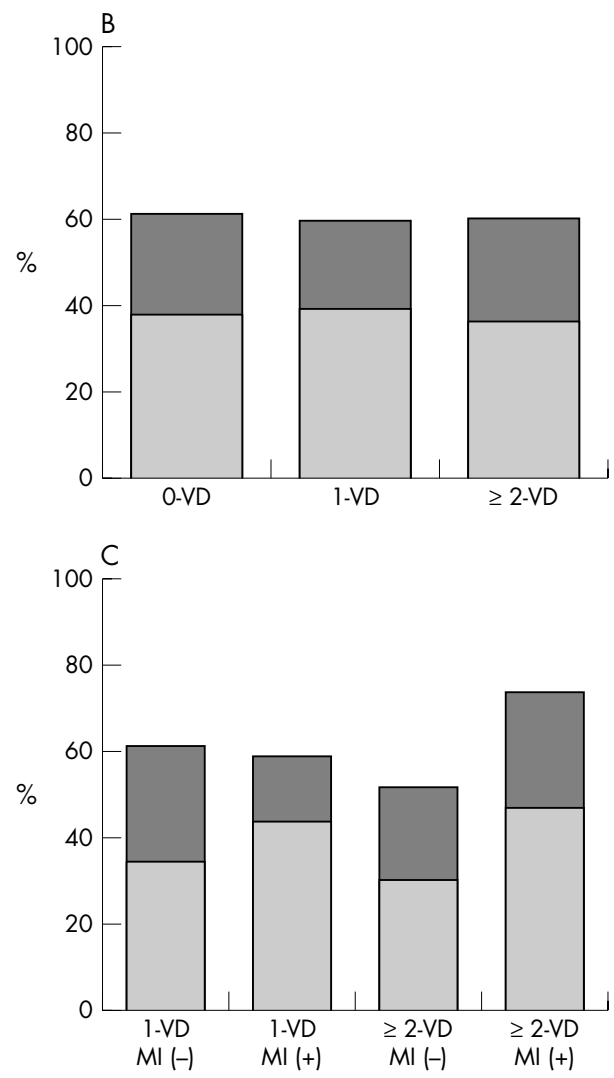

Figure 1 Frequency distribution during a $75 \mathrm{~g}$ oral glucose tolerance test (75g-oGT). (A) (Top) Frequency distribution of normal fasting glucose (NFG) and impaired fasting glucose (IFG) and (bottom) distribution of normal (NGT), impaired (IGT), and diabetic glucose tolerance (DGT) in all patients. The proportions of IGT (39\%) and DGT $(21 \%)$ were very high and only $40 \%$ of patients had NGT. The frequency distribution did not differ significantly by (B) the number of diseased coronary arteries $(0$ vessel disease (VD), 1-VD, or $\geqslant 2-V D$ ) or (C) the presence $(+)$ or absence $(-)$ of previous myocardial infarction (MI). The dark grey and light grey areas indicate DGT and IGT, respectively. 
patients with vasospastic angina pectoris and two patients who had no significant stenosis in coronary arteries with the clinical symptoms and signs of myocardial ischaemia. One patient who had a significant stenosis in the left main trunk was considered to have $\geqslant 2$-VD. In 1 -VD and $\geqslant 2$-VD, the patients were also divided into two groups: those who had no previous MI and those who had a previous MI. The previous MI was determined by the history of creatine kinase increase (more than twice the normal upper limit) or by the presence of abnormal Q waves in at least two ECG leads. This study protocol was in accordance with the Declaration of Helsinki and was approved by the institutional review board (Hamamatsu University School of Medicine, Hamamatsu, Japan). All patients gave informed consent.

\section{Statistical analysis}

All the data are expressed as the mean (SD) of indicated numbers. Categorical variables were compared between groups of patients by $\chi^{2}$ analyses. Differences between groups were examined by Kruskal-Wallis test followed by MannWhitney U test with Bonferroni correction. Linear regression analyses were performed to predict post-challenge hyperglycaemia and hyperinsulinaemia with the fasting PG and IRI concentrations, lipid parameters, body mass index (BMI), and blood pressure.

\section{RESULTS}

\section{Patient characteristics}

Table 1 shows the basic characteristics of patients classified according to the number of diseased coronary arteries. Patients with 1-VD and $\geqslant 2$-VD had more previous MI than did patients with 0 -VD. There were no differences between the groups in age, sex, BMI, proportions of current smokers, haemoglobin $\mathrm{A}_{\mathrm{lc}}$, blood pressure, total cholesterol, and triglyceride. However, high density lipoprotein cholesterol concentration was significantly lower in patients with $\geqslant 2$-VD than in patients with 0 -VD or l-VD. In the l-VD and $\geqslant 2$-VD groups, more patients were taking $\beta$ adrenergic blockers and angiotensin converting enzyme inhibitors or angiotensin II receptor blockers, but fewer were taking

Table 2 Comparison of glucose parameters of $75 \mathrm{~g}$ oral glucose tolerance test (75g-oGTT) in patients categorised according to the number of significantly stenosed coronary arteries

\begin{tabular}{llll}
\hline & 0-VD & 1-VD & $\geqslant 2$-VD \\
\hline PG-0 (mg/dl) & & & \\
All & $95.0(11.7)$ & $94.5(10.4)$ & $92.6(10.0)$ \\
MI (-) & NA & $94.5(10.4)$ & $92.8(8.9)$ \\
MI (+) & NA & $94.4(10.4)$ & $92.3(11.7)$ \\
PG-30 (mg/dl) & & & \\
All & $175.3(34.1)$ & $171.1(26.6)$ & $161.6(25.2)$ \\
Ml (-) & NA & $172.0(24.0)$ & $160.1(25.2)$ \\
MI (+) & NA & $170.3(29.0)$ & $163.8(25.5)$ \\
PG-60 (mg/dl) & & & \\
All & $191.7(50.6)$ & $194.8(43.5)$ & $185.0(45.9)$ \\
MI (-) & NA & $195.3(44.2)$ & $180.9(46.8)$ \\
MI (+) & NA & $194.4(43.5)$ & $191.4(45.0)$ \\
PG-120 (mg/dl) & & & \\
All & $154.9(54.1)$ & $156.6(54.1)$ & $161.8(42.4)$ \\
MI (-) & NA & $163.8(58.4)$ & $155.3(39.6)$ \\
MI (+) & NA & $150.0(49.7)$ & $171.6(45.7)$ \\
AUCglu (mg/dl/h) & $166.3(36.9)$ & $166.8(32.1)$ & $161.8(30.2)$ \\
All & NA & $169.0(32.5)$ & $158.3(28.8)$ \\
MI (-) & NA & $164.8(32.1)$ & $167.2(32.3)$ \\
MI (+) & & &
\end{tabular}

Data are mean (SD).

AUCglu, area under the glucose curve during $75 \mathrm{~g}$-oGT; NA, not applicable; PG, plasma glucose concentrations before (0) and after $(30$, 30 minutes; 60,60 minutes; 120,120 minutes) a $75 \mathrm{~g}$ oral glucose challenge. calcium channel blockers than in the 0-VD group. Most of the patients taking diuretics had $\geqslant 2-\mathrm{VD}$. Patients with 1-VD and $\geqslant 2$-VD also tended to take more lipid lowering agents.

\section{Glucose metabolism and CAD}

Figure 1A exhibits the frequency distributions of patients with normal $(76 \%)$ and impaired fasting glucose (24\%; top) concentrations and of patients with normal, impaired, and diabetic glucose tolerance at 120 minutes (bottom). The proportions of patients with impaired and diabetic glucose tolerance were very high $39 \%$ with impaired and $21 \%$ with diabetic glucose tolerance); only $40 \%$ had normal glucose tolerance. Among patients with normal fasting glucose, $48 \%$ had normal glucose tolerance, $37 \%$ had impaired glucose tolerance, and $15 \%$ had diabetic glucose tolerance. The corresponding proportions of patients with impaired fasting glucose were $12 \%, 47 \%$, and $41 \%$, respectively. Figure $1 \mathrm{~B}$ and fig $\mathrm{IC}$ show the frequency distributions of normal, impaired, and diabetic glucose tolerance among the patients grouped by number of diseased vessels and presence or absence of previous MI. The distributions were similar in the groups and did not differ significantly by the number of diseased coronary arteries or by the presence of previous MI. Table 2 provides more detailed analyses of glucose metabolism. The number of diseased coronary arteries or the presence of previous MI did not significantly alter PG and AUCglu during the $75 \mathrm{~g}$-oGTT.

\section{Insulin response and CAD}

Table 3 summarises IRI, HOMA-IR, AUCins, and the ratio of AUCins to AUCglu (AUCins:AUCglu) during 75g-oGTT. All

Table 3 Comparison of insulin parameters of $75 \mathrm{~g}$-oGTT in patients categorised according to the number of significantly stenosed coronary arteries

\begin{tabular}{|c|c|c|c|}
\hline & $0-V D$ & I-VD & $\geqslant 2-\mathrm{VD}$ \\
\hline \multicolumn{4}{|c|}{ IRI-0 ( $\mu \mathrm{U} / \mathrm{ml})$} \\
\hline All" & $8.1(5.0)$ & $8.8(4.4)$ & $9.7(5.5)$ \\
\hline MI (-) & NA & $7.8(3.4)$ & $8.6(4.5)$ \\
\hline MI (+) & NA & $9.7(5.1)$ & $11.4(6.5)$ \\
\hline \multicolumn{4}{|c|}{ IRI-30 ( $\mu \mathrm{U} / \mathrm{ml})$} \\
\hline All & $60.9(48.8)$ & $73.2(51.5)$ & $77.8(62.1)$ \\
\hline MI (-) & NA & $66.9(55.9)$ & 74.3 (71.0) \\
\hline MI (+) & NA & $78.8(55.8)$ & $83.1(46.7)$ \\
\hline \multicolumn{4}{|c|}{ IRI-60 ( $\mu \mathrm{U} / \mathrm{ml})$} \\
\hline All & $68.8(50.9)$ & $103.3(88.2)^{*}$ & $97.8(73.6)^{*}$ \\
\hline MI (-) & NA & $93.0(64.5)$ & $81.2(55.4)$ \\
\hline MI (+) & NA & $112.6(105.4)$ & $123.1(90.8)$ \\
\hline \multicolumn{4}{|c|}{ IRI-120 ( $\mu \mathrm{U} / \mathrm{ml})$} \\
\hline All & 73.5 (53.4) & $99.3(108.3)$ & 119.9 (133.7) \\
\hline MI (-) & NA & $105.3(140.1)$ & $88.3(52.3)$ \\
\hline Ml $(+)$ & NA & $93.9(69.6)$ & $168.2(195.8) \dagger$ \\
\hline \multicolumn{4}{|l|}{ HOMA-IR } \\
\hline All & $1.91(1.20)$ & $2.06(1.09)$ & $2.24(1.29)$ \\
\hline MI (-) & NA & $1.81(0.80)$ & $2.00(1.14)$ \\
\hline Ml $(+)$ & NA & $2.29(1.27)$ & $2.61(1.45)$ \\
\hline \multicolumn{4}{|c|}{ AUCins $(\mu \mathrm{U} / \mathrm{ml} / \mathrm{h})$} \\
\hline All & $60.4(41.3)$ & $82.9(55.7)^{*}$ & $87.3(65.7)^{*}$ \\
\hline MI (-) & NA & $78.9(62.2)$ & $72.2(41.3)$ \\
\hline Ml $(+)$ & NA & $86.6(49.9)$ & $110.4(87.6) \dagger$ \\
\hline \multicolumn{4}{|c|}{ AUCins:AUCglu $(\mu \mathrm{U} / \mathrm{mg})$} \\
\hline All & $0.37(0.21)$ & $0.51(0.35)^{*}$ & $0.54(0.37)^{*}$ \\
\hline MI (-) & NA & $0.48(0.38)$ & $0.46(0.26)$ \\
\hline MI (+) & NA & $0.54(0.32)$ & $0.66(0.48) \dagger$ \\
\hline
\end{tabular}

Data are mean (SD).

${ }^{*} p<0.05 v 0-V D ;+p<0.05 v \geqslant 2-V D$ and $\mathrm{Ml}(-)$ by Kruskal-Wallis test followed by Mann-Whitney $U$ test with Bonferroni correction. AUCins, area under the insulin curve in $75 \mathrm{~g}-\mathrm{oGT} ; \mathrm{HOMA}-\mathrm{IR}$, insulin resistance estimated by homeostasis model assessment; IRI, plasma insulin concentrations before $(0)$ and after $(30,30$ minutes; 60,60 minutes; 120, 120 minutes) the ingestion of a $75 \mathrm{~g}$ oral glucose challenge. 

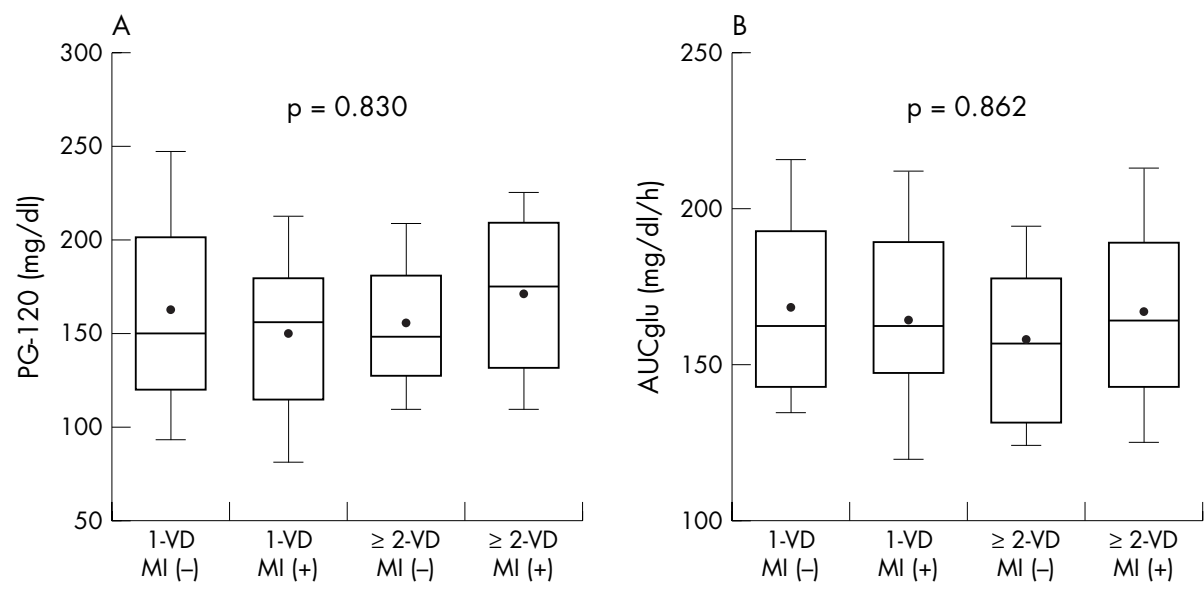

Figure 2 Box and whisker plots of (A) plasma glucose concentration 120 minutes after $75 \mathrm{~g}-\mathrm{o} G T$, (B) area under the glucose curve (AUCglu), (C) plasma insulin concentration 120 minutes affer $75 \mathrm{~g}-\mathrm{oGTT}$ (IRI-120), and (D) area under the insulin curve (AUCins). Each plot indicates the mean $(\cdot)$, median (middle bar in rectangle), and 10th (bottom of bar), 25th (bottom of rectangle), 75 th (top of rectangle), and 90 th centiles (top of bar) in patients with $1-V D$ and $\geqslant 2$-VD. Patients were also divided into $M I(-)$ and $M I(+)$ groups. The MI (+) group with $\geqslant 2$-VD had higher insulin concentrations, whereas glucose concentrations were similar in all groups. ${ }^{*} \mathrm{p}<0.05 \vee \mathrm{MI}(-)$ in 1-VD; tp $<0.05 \vee \mathrm{Ml}(-)$ in $\geqslant 2$-VD by Kruskal-Wallis test followed by MannWhitney $\mathrm{U}$ test with Bonferroni
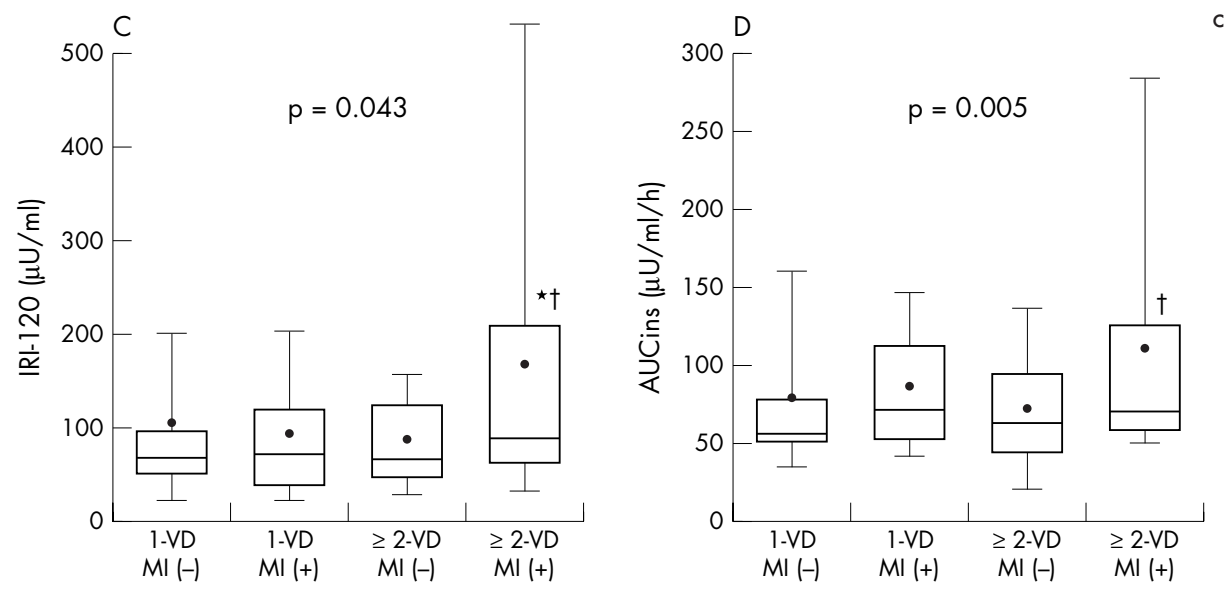

the values tended to increase with an increase in the number of diseased coronary arteries. The values of IRI-60, AUCins, and AUCins:AUCglu were significantly higher in 1-VD and $\geqslant 2$-VD than in 0 -VD. However, there were no significant differences between patients with $1-\mathrm{VD}$ and patients with $\geqslant 2$-VD. Next, to examine the relation between the insulin response and a previous MI, the insulin concentrations were compared between patients with and without a previous MI and with $1-V D$ and $\geqslant 2$-VD. IRI tended to be higher in the presence of previous MI and the difference was significant in IRI-120, AUCins, and AUCins:AUCglu.

Figure 2 shows the box and whisker plots of PG-120, AUCglu, IRI-120, and AUCins. Patients with $\geqslant 2$-VD and a previous MI had very high post-challenge insulin concentrations, whereas the distribution of glucose did not differ between groups.

\section{Prediction of post-challenge hyperglycaemia and hyperinsulinaemia}

Since the patients with CAD had a high prevalence of abnormal glucose tolerance and post-challenge hyperinsulinaemia was associated with the number of diseased coronary arteries and the presence of previous MI, it became important to predict the post-challenge glucose and insulin concentrations with other fasting metabolic parameters. Table 4 shows the linear regression analyses for all patients. There was no significant relation between IRI-120 and fasting PG, haemoglobin $\mathrm{A}_{\mathrm{lc}}$, lipid parameters, or blood pressure, whereas

\begin{tabular}{|c|c|c|c|c|}
\hline \multirow{2}{*}{$\begin{array}{l}\text { Fasting metabolic } \\
\text { parameter }\end{array}$} & \multicolumn{2}{|l|}{ PG-120 } & \multicolumn{2}{|l|}{ IRI-120 } \\
\hline & Regression coefficient (SE) & p Value & Regression coefficient (SE) & $\mathrm{p}$ Value \\
\hline PG-0 & $1.94(0.37)$ & $<0.001$ & $-0.70(0.87)$ & 0.42 \\
\hline IRI-0 & $1.55(0.84)$ & 0.07 & $12.55(1.53)$ & $<0.001$ \\
\hline HOMA-IR & $8.83(3.49)$ & 0.01 & $47.72(6.68)$ & $<0.001$ \\
\hline TC & $-0.04(0.11)$ & 0.70 & $0.20(0.25)$ & 0.43 \\
\hline HDL-C & $-0.29(0.33)$ & 0.37 & $-0.94(0.71)$ & 0.19 \\
\hline TG & $0.07(0.05)$ & 0.24 & $0.01(0.11)$ & 0.94 \\
\hline $\mathrm{HbA}_{1 \mathrm{c}}$ & $43.07(9.69)$ & $<0.001$ & $15.11(22.70)$ & 0.51 \\
\hline BMI & $4.78(1.40)$ & $<0.001$ & $14.32(2.95)$ & $<0.001$ \\
\hline SBP & $0.25(0.19)$ & 0.19 & $0.52(0.42)$ & 0.21 \\
\hline DBP & $-0.10(0.75)$ & 0.64 & $-0.45(0.67)$ & 0.50 \\
\hline
\end{tabular}


fasting $P G$ and haemoglobin $A_{1 c}$ were correlated with PG-120. Only HOMA-IR and BMI were associated with both PG-120 and IRI- 120.

\section{DISCUSSION}

This study indicated that, firstly, patients with CAD without a previous diagnosis of DM had a high prevalence of abnormal glucose tolerance; secondly, post-challenge hyperinsulinaemia was associated with the number of diseased coronary arteries and the presence of previous MI, whereas the parameters of glucose response were not; and thirdly, fasting PG, haemoglobin $A_{1 c}$, and lipid parameters could not predict post-challenge hyperinsulinaemia.

\section{Glucose metabolism and CAD}

In this study, only $40 \%$ of patients had normal glucose tolerance and $21 \%$ had newly detected diabetes. This high prevalence was apparent despite the exclusion of patients with previously diagnosed $\mathrm{DM}$ or $\mathrm{PG} \geqslant 126 \mathrm{mg} / \mathrm{dl}$ ( $7.0 \mathrm{mmol} / \mathrm{l})$. A recent Swedish study also found that 35\% of patients with acute MI had impaired glucose tolerance and $31 \%$ had newly diagnosed DM. ${ }^{13}$ In Japanese people aged 60 years, the prevalence of abnormal glucose tolerance was found to be $20 \%$, which was similar to that in Swedish people. ${ }^{314}$ Therefore, it is highly likely that there is a high prevalence of abnormal glucose tolerance in patients with CAD.

The American Diabetes Association recommended that the fasting PG alone should be used to diagnose DM. ${ }^{12}$ However, the DECODA study showed that more than half of diabetic patients had isolated post-challenge hyperglycaemia and three quarters of patients with impaired glucose tolerance had normal fasting glucose. ${ }^{2}$ In fact, in this study $71 \%$ (41 of 57 ) of patients with impaired glucose tolerance and 52\% (16 of 30) of patients with diabetic glucose tolerance were categorised as having normal fasting glucose. Since several large cohort studies have indicated that people with prediabetic conditions have a raised risk of cardiovascular diseases, identification of these patients is very important. ${ }^{13} 15$

In this study, there was no correlation between the number of diseased coronary arteries or the presence of previous MI and the frequency distribution of glucose tolerance or various parameters of glucose response during $75 \mathrm{~g}$-oGTT. Takezako and colleagues ${ }^{10}$ also reported that the glucose response did not correlate with the severity of coronary atherosclerosis. On the other hand, Kanauchi and colleagues ${ }^{16}$ described that post-challenge hyperglycaemia was independently associated with the number of stenosed coronary arteries in nondiabetic patients. ${ }^{16}$ The reason for this difference is unknown but the numbers of patients in those studies were relatively small. Our study may clarify this issue in a larger group of patients without a previous diagnosis of DM.

\section{Insulin response and CAD}

The euglycaemic hyperinsulinaemic clamp technique (glucose clamp) has been used as the best method for evaluating insulin resistance. However, its technical difficulty has limited its clinical application especially in cardiovascular departments. Therefore, we evaluated glucose and insulin responses to the $75 \mathrm{~g}$-oGTT as a simple alternative to the glucose clamp test. In this study, IRI-60 and IRI-120, but not IRI-0 or HOMA-IR, were associated with the number of diseased coronary arteries and the presence of previous MI. When the analyses were limited to age matched male patients, the results were quite similar (data not shown). We cannot define the reason but this finding indicates the importance of evaluating the insulin response to the glucose challenge test.
Although post-challenge hyperinsulinaemia does not necessarily reflect insulin resistance, our finding was very consistent with previous studies that showed the relation between hyperinsulinaemia and the severity of CAD. ${ }^{10}$ Insulin resistance with hyperinsulinaemia accelerates atherosclerosis both directly through effects of insulin on the arterial wall and indirectly through a clustering of major cardiovascular risk factors that are part of the insulin resistance syndrome. ${ }^{5717}$ However, we also have to consider the possibility that the different medications among the groups affected the differences in insulin response. Thus, compared with patients with 0 -VD, significantly more patients with $1-\mathrm{VD}$ and $\geqslant 2$-VD were taking $\beta$ adrenergic blockers and angiotensin converting enzyme inhibitors or angiotensin II receptor blockers but fewer were taking calcium channel blockers. More patients with I-VD and $\geqslant 2$-VD also tended to take lipid lowering agents. Most of the patients taking diuretics had $\geqslant 2$-VD.

The statistical analyses showed that hyperinsulinaemia correlated more with the presence of previous MI than with the number of diseased coronary arteries. Although the analysed data on relatively large MI were limited, this finding may point to a possible relation between hyperinsulinaemia and vasoconstriction or thrombus formation. In fact, Hedblad and colleagues ${ }^{18}$ showed in non-diabetic patients that insulin resistance was associated with an increased incidence of MI and death. Insulin is predominantly an endothelium dependent vasodilator. ${ }^{7}$ In patients with insulin resistance, however, chronic hyperinsulinaemia causes coronary vasoconstriction by increasing the release of endothelin- 1 and sympathetic nerve activity and by reducing the endothelial response to vasodilators. Insulin resistance with hyperinsulinaemia is also associated with several dysregulations of coagulation and fibrinolysis. For example, increased expression and secretion of plasminogen activator inhibitor-1 by hepatocytes and endothelial cells and increased concentrations of von Willebrand factor and fibrinogen were reported. These disorders may be due to secretion of several adipocytokines including interleukin- 6 and tumour necrosis factor $\alpha{ }^{19}$

In this study, none of the conventional risk factors except for BMI predicted post-challenge hyperinsulinaemia. The result was similar even when only male patients were analysed (data not shown). This may be because patients were already being treated for each risk factor but it also suggests the importance of measuring insulin concentrations directly and to focus future interventions on the underlying abnormality.

\section{Conclusion}

Among patients with $\mathrm{CAD}$, the proportions with impaired and diabetic glucose tolerance during 75g-oGTT were very high. Post-challenge hyperinsulinaemia rather than hyperglycaemia was associated more with the number of diseased coronary arteries and the presence of previous MI. The insulin response to the glucose challenge test requires further investigation as a potential risk factor for CAD and a potential target for intervention.

\section{Authors' affiliations \\ H Satoh, H Terada, A Uehara, H Katoh, M Matsunaga, K Yamazaki, F Matoh, H Hayashi, Division of Cardiology, Internal Medicine III, Hamamatsu University School of Medicine, Hamamatsu, Japan}

\section{REFERENCES}

1 DECODE Study Group. Glucose tolerance and mortality: comparison of WHO and American Diabetes Association diagnostic criteria. The DECODE study group. Lancet 1999;354:617-21. 
2 Qiao Q, Nakagami T, Tuomilehto J, for the International Diabetes Epidemiology Group, et al. DECODA Study Group. Comparison of the fasting and the 2- $h$ glucose criteria for diabetes in different Asian cohorts. Diabetologia 2000:43:1470-5.

3 Tominaga $M$, Eguchi $\mathrm{H}$, Manaka $\mathrm{H}$, et al. Impaired glucose tolerance is a risk factor for cardiovascular disease, but not impaired fasting glucose. The Funagata diabetes study. Diabetes Care 1999;22:920-4.

4 UKPDS Group. Intensive blood-glucose control with sulphonylureas or insulin compared with conventional treatment and risk of complications in patients with type 2 diabetes (UKPDS 33). UK Prospective Diabetes Study (UKPDS) Group. Lancet 1998;352:837-53.

5 Despres JP, Lamarche B, Mauriege P, et al. Hyperinsulinemia as an independent risk factor for ischemic heart disease. N Engl J Med 1996;334:952-7.

6 Pyorala M, Miettinen H, Laakso M, et al. Hyperinsulinemia and the risk of stroke in healthy middle-aged men: the 22-year follow-up results of the Helsinki policemen study. Stroke 1998;29:1860-6

7 Sundell J, Knuuti J. Insulin and myocardial blood flow. Cardiovasc Res 2003:57:312-9.

8 Onat A, Ceyhan K, Sansoy V, et al. Fasting insulin levels independently associated with coronary heart disease in non-diabetic Turkish men and women. Int J Cardiol 2002;86:61-9.

9 Bressler P, Bailey SR, Matsuda M, et al. Insulin resistance and coronary artery disease. Diabetologia 1996:39:1345-50.

10 Takezako T, Saku K, Zhang B, et al. Insulin resistance and angiographical characteristics of coronary atherosclerosis. Jpn Circ J 1999;63:666-73.
11 Matthews DR, Hosker JP, Rudenski AS, et al. Homeostasis model assessment: insulin resistance and beta-cell function from fasting plasma glucose and insulin concentrations in man. Diabetologia 1985;28:412-9.

12 The Expert Committee on the Diagnosis and Classification of Diabetes Mellitus. Follow-up report on the diagnosis of diabetes mellitus. The Expert Committee on the Diagnosis and Classification of Diabetes Mellitus. Diabetes Care 2003;26:3160-7.

13 Norhammar A, Tenerz A, Nilsson G, et al. Glucose metabolism in patients with acute myocardial infarction and no previous diagnosis of diabetes mellitus: a prospective study. Lancet 2002:359:2140-4.

14 Lindahl B, Weinehall L, Asplund K, et al. Screening for impaired glucose tolerance: results from a population-based study in 21,057 individuals. Diabetes Care 1999;22:1988-92.

15 Shaw JE, Hodge AM, de Courten $M$, et al. Isolated post-challenge hyperglycaemia confirmed as a risk factor for mortality. Diabetologia 1999;42:1050-4

16 Kanauchi M, Tsujimoto N, Hashimoto T. Advanced glycation end products in nondiabetic patients with coronary artery disease. Diabetes Care 2001;24:1620-3.

17 Howard G, O'Leary DH, Zaccaro D, et al. Insulin sensitivity and atherosclerosis. The Insulin Resistance Atherosclerosis Study (IRAS) Investigators. Circulation 1996;93:1809-17.

18 Hedblad B, Nilsson P, Engstrom G, et al. Insulin resistance in non-diabetic subjects is associated with increased incidence of myocardial infarction and death. Diabet Med 2002;19:470-5.

19 Yudkin JS. Abnormalities of coagulation and fibrinolysis in insulin resistance. Diabetes Care 1999;22(suppl 3):C25-30.

\section{IMAGES IN CARDIOLOGY}

\section{Massive air embolism after Maze}

doi: 10.1136/hrt.2004.04390

A 66 year old white man underwent mitral valve replacement in another city for severe mitral regurgitation. Surgery included a Maze procedure due to persistent atrial fibrillation. The postoperative course was uncomplicated. Two weeks after discharge the patient collapsed. Being unconscious he had to be intubated. An initial computed tomographic (CT) scan and magnetic resonance imaging of the head was normal. A transoesophageal echocardiogram showed good function of the prosthetic valve. The patient developed severe sepsis. In a control CT scan two weeks later multiple intracerebral air emboli as well as multiple cerebral infarctions were observed (panels A and $\mathrm{B})$. A CT scan of the thorax and the abdomen revealed a thrombus and free air in the left atrium (panel C) as well as embolic lesions in both kidneys and the spleen. Finally, an oesophagoatrial fistula was diagnosed by oesophagoscopy and palliative stenting was performed. The patient died in septic shock with the neurological picture of an acinetic mutism. To our knowledge this is the first case reporting multiple air emboli caused by an oesophagoatrial fistula after cardiac surgery involving the Maze procedure. In case of postoperative sepsis and neurological deficits one should think of this form of complication.
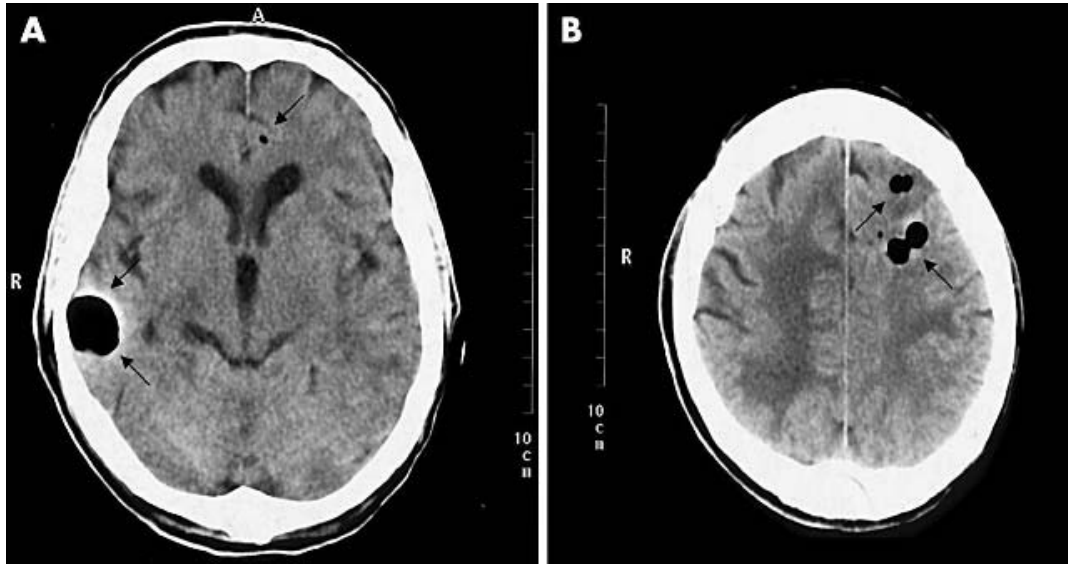

Cerebral computed tomographic (CT) scan showing multiple air emboli (arrows).
A Zirlik

T K Nordt azirlik@rics.bwh.harvard.edu

Thoracic CT scan revealing free air (1) and a large thrombus (2) in the left atrium. Ao, aorta; $\mathrm{E}$, oesophagus; LA, left atrium; LV, left ventricle; $\mathrm{RV}$, right ventricle. 